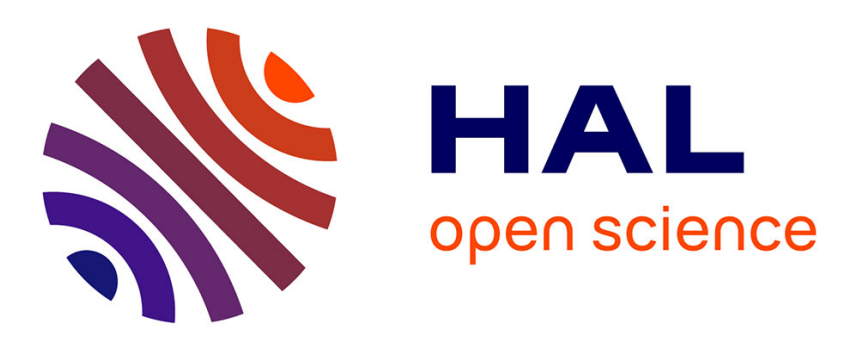

\title{
Extended Receive Spatial Modulation MIMO scheme for Higher Spectral Efficiency
}

Ali Mokh, Jean-Christophe Prévotet, Matthieu Crussière

\section{To cite this version:}

Ali Mokh, Jean-Christophe Prévotet, Matthieu Crussière. Extended Receive Spatial Modulation MIMO scheme for Higher Spectral Efficiency. 2018 IEEE 87th Vehicular Technology Conference, Jun 2018, Porto, Portugal. 10.1109/vtcspring.2018.8417598 . hal-01722208

\section{HAL Id: hal-01722208 \\ https://hal.science/hal-01722208}

Submitted on 3 Mar 2018

HAL is a multi-disciplinary open access archive for the deposit and dissemination of scientific research documents, whether they are published or not. The documents may come from teaching and research institutions in France or abroad, or from public or private research centers.
L'archive ouverte pluridisciplinaire HAL, est destinée au dépôt et à la diffusion de documents scientifiques de niveau recherche, publiés ou non, émanant des établissements d'enseignement et de recherche français ou étrangers, des laboratoires publics ou privés. 


\title{
Extended Receive Spatial Modulation MIMO scheme for Higher Spectral Efficiency
}

\author{
Ali Mokh, Maryline Hélard, Matthieu Crussière \\ Univ Rennes, INSA Rennes, IETR, CNRS, UMR 6164, F-35000 Rennes
}

\begin{abstract}
Spatial modulation (SM) schemes use the index of the transmit (resp. the receive) antenna to transmit additional data, and therefore allow for a new exploitation of the space dimension to increase the spectral efficiency of MIMO systems. The baseline of the proposed study relies on the so-called extended receive antenna shift keying (ERASK) concept consisting in transmitting the spatial bits by focusing the transmit energy towards subsets of receive antennas. With ERASK, the subsets of targeted antennas can be of any size leading to an amount of additional spatial bits directly equal to the number of receive antennas. However, an ON/OFF keying is used to carry the spatial symbols meaning that a zero power level is associated for non targeted antennas. Consequently, the transmission of additional IQ symbols in ERASK is not compatible with every spatial symbol configurations. In this paper, we propose a novel scheme referred to as Extended RSM (ERSM) that sends additional IQ modulated signals in ERASK, in order to increase the overall spectral efficiency. The key idea is to associate a non zero power levels for all spatial symbols. Two power levels are used to transmit the spatial and the IQ symbols, and an optimal ratio of the power is calculated. The theoretical performance of the proposed ERSM scheme is derived and then validated through simulations.
\end{abstract}

Index Terms-Receive Spatial Modulation, Receive Antenna Shift Keying, MIMO, Phase Shift Keying, Zero Forcing

\section{INTRODUCTION}

Today, multiple-input and multiple-output (MIMO) wireless systems represent one of the major steps in the enhancement of many wireless communication systems [1], thanks to their ability to allow for an impressive increase in system capacity [2]. A new MIMO scheme called spatial modulation (SM) appeared in the early 2000s, that consists in exploiting the index of transmit or receive antennas to convey additional information bits. In such techniques, location-dependent spatial information is utilized to carry additional information bits thereby boosting the overall spectral efficiency (SE) [3][4].

The general idea behind SM is to transmit one block of information bits in two parts by means of two complementary mapping functions. One of them consists of classical bits to IQ-symbols mapping of an M-order modulation. The other relies on associating the remaining bits, commonly referred to as "spatial bits", to a unique antenna index chosen from a set of transmit antennas [5]. In [6], the authors extend the concept of the SM by allowing more than one transmit antenna to be active at every channel use, and so to increase the number of different combinations of multiple active antennas. The modulation scheme is referred to as Generalized SM (GSM).

The same concept of SM-MIMO has been applied to the receiver, called Receive-Spatial Modulation (RSM) [7] [8], or Receive Antenna Shift Keying (RASK) [9][10], where the index of the targeted receive antenna (RA) carries the additional spatial information, using a pre-processing technique to target the RAs. A generalization of the RSM principle, further referred to as GPSM (Generalised Pre-coding aided Spatial Modulation), is proposed in [11] where the transmit antennas (TA) concentrate the signal energy towards a fixed and constant number of RAs to increase the SE. Opposed to conventional SM where a subset of RF chains is deployed, here all TAs and RAs are active. Finally, an extended model of RASK is introduced in [12], referred to as ERASK, where all possible combinations of targeted RAs are used, even that one where zero RA is targeted, i.e. no signal is emitted. Among other candidates, this last scheme offers the highest spectral efficiency (SE) at the level of the spatial bits, i.e. equal to the number of RAs [13][14][15].

As explained at the very beginning of the introduction, SM techniques are expected to offer an additionnal means to transmit bits in superposition to the conventional IQ bits in MIMO systems. In this paper, we investigate how spatial bits and IQ bits can simultaneously be sent using the ERASK strategy. As detailed in the paper, the transmission of IQ bits upon spatial bits is not straightforward in ERASK due to the variable number of targeted RAs at each symbol duration. Inspired by the dual-layered transmission (DLT) philosophy introduced in [16], we propose a novel scheme referred to as Extended RSM (ERSM) which allows a variable number of RAs to be targeted while simultaneously sending IQ bits. DLT is based on transmitter pre-coding (TPC) and turns out to exploit SM systems at its full spatial multiplexing capability. With DLT, all the receive antennas are targeted by IQ signals but using two different power levels to code the spatial information. Similarly, the proposed ERSM system relies on the use of two non zero power levels to send additional IQ data. In this paper, we demonstrate how ERSM provides a SE equal to $N_{r}+\log _{2}(M)$, where $N_{r}$ is the number of receive antennas, and $M$ is the order of modulation. We also derive the theoretical performance for the scheme which are corroborated by simulations.

The rest of the paper is organized as follows. In Section II, we present the system model and we provide a review of ERASK schemes with a description of its block diagrams. In Section III, we present the principle of the ERSM scheme, we explain the transmission of a sequence of bits, in order to show how the spectral efficiency is increased, and we detail the block diagram of the ERSM scheme. The theoretical 
computation of the Bit Error Rate of ERSM is detailed in Section IV, with the derivation of the optimal power levels. Simulation results are provided in Section V, and a conclusion is drawn in Section VI.

Notation: Boldface is used for matrices $\mathbf{A} \cdot \mathbf{A}^{T}, \mathbf{A}^{\dagger}, \operatorname{Tr}(\mathbf{A})$ respectively denotes the transpose, the conjugate transpose and the trace of matrix A. $\Re\{$.$\} denotes the real part of a$ complex value. $\|$.$\| denotes the Euclidean norm of a vector or$ a matrix. $\mathbb{E}\{$.$\} is the expectation operator. T_{i}$, and $R_{j}$ denotes respectively the $i$-th TA and the $j$-th RA.

\section{Overview of Extended Receive Antenna Shift KEYING}

\section{A. System Model}

A MIMO system with $N_{t}$ TAs and $N_{r}$ RAs is considered, where $N_{t}>N_{r}$. Assuming a flat fading channel between the transmitter and the receiver, the receive signal vector can be written as:

$$
\mathbf{Y}=\mathbf{H} . \mathbf{S}+\mathbf{N}
$$

where $\mathbf{H} \in \mathbb{C}^{N_{r} \times N_{t}}$ is the MIMO channel matrix with element $H_{j, i}$ representing the complex channel coefficient between $i$ th antenna at the transmitter $T_{i}$ and the $j$ th antenna at the receiver $R_{j} . \mathbf{Y} \in \mathbb{C}^{N_{r} \times 1}$ is the vector of the received signals on all RAs, $\mathbf{S} \in \mathbb{C}^{N_{t} \times 1}$ is the vector of the transmitted signals by all TAs, and $\mathbf{N} \in \mathbb{C}^{N_{r} \times 1}$ is the vector of additive white Gaussian noise (AWGN) samples $\eta_{j}$ at antenna $R_{j}$ such that $\eta_{j} \sim \mathcal{C N}\left(0, \sigma_{n}^{2}\right)$.

\section{B. Extended RASK principles}

With the basic RASK scheme, information is transmitted by means of spatial bits such that only one RA during is targeted during each symbol duration. This leads to a SE of $\log _{2}\left(N_{r}\right)$ [9]. Such SE can be increased up to $N_{r}$ by the use of the Extended RASK (ERASK) scheme which allows $0 \leq N_{a} \leq N_{r}$ antennas to be simultaneously targeted [12]. At the transmitter side, a group of $\beta_{s}=N_{r}$ bits is mapped onto a spatial symbol $\mathbf{X} \in \mathbb{N}^{N_{R} \times 1}$ which is written as:

$$
\mathbf{X}=\left[\begin{array}{lllll}
x_{1} & \ldots & x_{j} & \ldots & x_{N_{C D}}
\end{array}\right]^{T}
$$

In a basic approach, a simple ON/OFF keying is used to transmit the spatial symbols, where the non targeted antennas is allocated with a zero power level and the total transmitted power is shared so as to focus on the selected RAs. More precisely, $x_{j}=\{0,1\}$ depending on whether $R_{j}$ is targeted or not. A ZF pre-processing is performed in order to focus the signal towards the targeted antennas, where the pseudoinverse of the channel matrix $\mathbf{W} \in \mathbb{C}^{N_{t} \times N_{r}}$, is used as a pre-filter:

$$
\mathbf{W}=\mathbf{H}^{\dagger}\left(\mathbf{H} \cdot \mathbf{H}^{\dagger}\right)^{-1}
$$

\section{Extended Receive Spatial Modulation PROPOSAL}

\section{A. ERSM Principles}

The idea behind the proposed extended RSM (ERSM) consists in merging conventionnal IQ modulation based on Phase Shift Keying (PSK) and the Extended RASK concept previously presented. Hence, at each symbol duration, each RA is targeted with one among two power levels $\left\{P_{1}, P_{2}\right\}$ and receives the same IQ symbol. In this scheme, the number $M_{1}$ of possible spatial symbols is equal to $2^{N_{r}}$, providing a transmission of $\beta_{s}=N_{r}$ spatial bits. Consequently, the total SE of ERSM is:

$$
\beta=\beta_{s}+\beta_{M}
$$

where $\beta_{M}=\log _{2}(M), \mathrm{M}$ is the order of an M-PSK modulation. Fig. 1 provides an illustration of the ERSM scheme concept with $N_{r}=2$ and a BPSK modulation, resulting a SE of $\beta=3 \mathrm{bits} / \mathrm{s} / \mathrm{Hz}$. The signal with power $P_{1}$ (resp. $P_{2}$ ) is represented in yellow (resp. blue). The BPSK symbols are represented by their phase: 0 or $\pi$. Steps 1 and 2 allow for forming a super symbol transmitting 3 bits during each symbol duration. The spatial mapping used in this example is the following:

- $P_{1}$ is allocated to targeted antenna to transmit ' 0 ',

- $P_{2}$ is allocated to targeted antenna to transmit ' 1 '.

The two power levels must be carefully selected to minimize the total error probability, so let us define:

$$
\alpha=\frac{P_{2}}{P_{1}}
$$

with $P_{1}<P_{2}$ (i.e. $\alpha>1$ ). In order to have an average total transmit power equal to 1 , we have:

$$
P_{1}=\frac{2}{(1+\alpha) N_{r}}
$$

and

$$
P_{2}=\frac{2 \alpha}{(1+\alpha) N_{r}}
$$

In the following, an optimal value for $\alpha$ is calculated. The transmitted signal also depends on an additional bit related to a BPSK modulation. Hence, at step 3, the pre-processing is performed at the transmitter so as to create a beam to concentrate the allocated energy towards the targeted antennas. At step 4, the receiver estimates the phase of the sum of all signals in order to demodulate the BPSK bit, and after it estimates which power level was allocated by analyzing the amount of received signal amplitude at each antenna, to deduce the transmitted spatial symbol.

\section{B. Block Diagram of ERSM}

The block diagram of the ERSM system is depicted in Fig. 2. A group of $\beta$ bits is mapped onto a super-symbol vector $\mathbf{X} \in \mathbb{C}^{N_{r} \times 1}$ :

$$
\mathbf{X}=\left[x_{m}^{k}\right]^{T}=\left[\begin{array}{llll}
x_{m}^{k_{1}} & x_{m}^{k_{2}} & \ldots & x_{m}^{k_{N_{r}}}
\end{array}\right]^{T}
$$




\begin{tabular}{|c|c|c|c|c|}
\hline 000101100111 & Symbol 1 & Symbol 2 & Symbol 3 & Symbol 4 \\
\hline 1) Segmentation & 000 & 101 & 100 & 111 \\
\hline $\begin{array}{l}\text { 2) Spatial \& PSK } \\
\text { mapping }\end{array}$ & $\left(-\sqrt{p_{1}},-\sqrt{p_{1}}\right)$ & $\left(\sqrt{p_{2}}, \sqrt{p_{1}}\right)$ & $\left(-\sqrt{p_{2}},-\sqrt{p_{1}}\right)$ & $\left(\sqrt{p_{2}}, \sqrt{p_{2}}\right)$ \\
\hline \multirow[t]{2}{*}{$\begin{array}{l}\text { 3) Preprocessing } \\
\text { (focalisation) }\end{array}$} & $\pi \pi$ & & & \\
\hline & $\nabla \nabla$ & & & $\nabla \nabla$ \\
\hline 4) Detection & $\left(-\sqrt{p_{1}},-\sqrt{p_{1}}\right)$ & $\left(\sqrt{p_{2}}, \sqrt{p_{1}}\right)$ & $\left(-\sqrt{\boldsymbol{p}_{2}},-\sqrt{\boldsymbol{p}_{1}}\right)$ & $\left(\sqrt{p_{2}}, \sqrt{p_{2}}\right)$ \\
\hline 5) Demapping & 000 & 101 & 100 & 111 \\
\hline
\end{tabular}

Figure 1. Example of the Extended-RSM system with $N_{r}=2$ using BPSK modulation

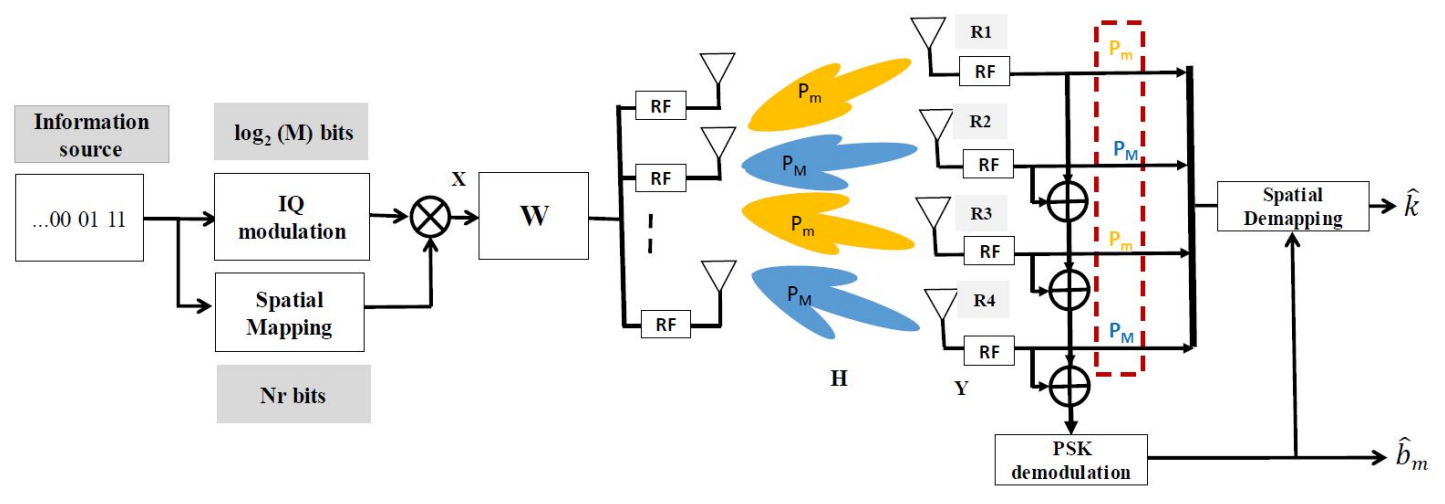

Figure 2. Block diagram of ERSM system

where $m \in 1, \ldots, M$ is a symbol taken from an $M$ order modulation and $k$ denotes for the spatial symbol. The mapping is made of two stages, one for the IQ symbol and another one for the spatial symbol. An M-PSK modulation symbol in the form of $b_{m}=e^{i \theta_{m}}$ is first constructed, depending on the bit stream to transmit. Then, the spatial symbol is mapped by allocating different power levels to the targeted RAs in applying the power allocation vector $P_{k}$ :

$$
x_{m}^{k}=\mathbf{P}_{k} b_{m}
$$

with

$$
\mathbf{P}_{k}=\left[\begin{array}{llll}
\sqrt{p_{1, k}} & \sqrt{p_{2, k}} & \cdots & \sqrt{p_{N_{r}, k}}
\end{array}\right]^{T}
$$

where $p_{i, k}, i \in\left[1, N_{r}\right]$ are taken from the set $\left\{P_{1}, P_{2}\right\}$ according to the spatial symbol $\mathrm{k}$.

Then, the pre-processing block transforms the vector of spatial symbols $\mathbf{X}$ into a vector of transmitted signals denoted by $\mathbf{S} \in \mathbb{C}^{N_{t} \times 1}$ using the ZF pre-processing matrix as defined by Eq.(3):

$$
\mathbf{S}=f . \mathbf{W} \cdot \mathbf{X}
$$

where

$$
f=\frac{1}{\sqrt{\sigma_{x}^{2} \operatorname{Tr}\left(\mathbf{W} \cdot \mathbf{W}^{H}\right)}}
$$

is a normalization factor used to guarantee that the average total transmit power $\bar{P}_{t}$ is equal to 1 . Since $\mathbf{X}$ has i.i.d. entries, the variance $\sigma_{x}^{2}=\mathbb{E}_{x}\left[x_{j} x_{j}^{*}\right]$ is independent of $j$ and comes in factor of the trace computation. Then, each entry of $\mathbf{X}$ is of amplitude $\sqrt{P_{1}}$ or $\sqrt{P_{2}}$ with a probability that can be easily verified to be $\frac{1}{2}$, leading to $\sigma_{x}^{2}=\frac{P_{1}+P_{2}}{2}$.

At the receiver, it is straightforward to obtain the expression of the receive signals:

$$
\mathbf{Y}=f \cdot \mathbf{X}+\mathbf{N}
$$

At the level of the RA $R_{j}$, the received signal then simply writes:

$$
\begin{aligned}
y_{j, k} & =f \times x_{m}^{j}+\eta_{j} \\
& =f \sqrt{p_{j, k}} \times b_{m}+\eta_{j}
\end{aligned}
$$

An M-PSK demodulation is operated to detect the IQ symbol transmitted and a spatial detection based on the level of the received amplitude at each RA is used to complete the whole transmit symbol detection. First, all received signals are summed up, and an M-PSK is performed to estimates the transmitted IQ symbol. We define $z_{k}$ the sum of the received signals when sending the spatial symbol $k$ :

$$
z_{k}=\sum_{j=1}^{N_{r}} y_{j, k}=\left(\sum_{j=1}^{N_{r}} \sqrt{p_{j, k}}\right) f b_{m}+\sum_{j=1}^{N_{r}} \eta_{j} .
$$

The estimated IQ symbol $\tilde{b}_{m}$ from the demodulation of $z_{k}$ is used to compensate the phase of all received signals in order 
to detect the spatial symbol. Accordingly, the receiver at $R_{j}$ compares the compensated received signal to a predefined threshold $\nu$ :

$$
p_{j, k}= \begin{cases}P_{1}, & \text { if } \Re\left\{y_{j} / \tilde{b}_{m}\right\} \leq \nu, \\ P_{2}, & \text { if } \Re\left\{y_{j} / \tilde{b}_{m}\right\} \geq \nu .\end{cases}
$$

The optimal threshold is easily deduced from:

$$
\nu=\mathbb{E}\left\{\frac{\Re\left\{y_{j, 1} / \tilde{b}_{m}\right\}+\Re\left\{y_{j, 2} / \tilde{b}_{m}\right\}}{2}\right\}=\frac{f\left(\sqrt{P_{1}}+\sqrt{P_{2}}\right)}{2} .
$$

with $y_{j 1}$ (resp. $y_{j 2}$ ) the receive signal on antenna $R_{j}$ if this antenna is targeted with the power level $P_{1}$ (resp. targeted by the power level $P_{2}$ ). Note that such a threshold can in practice be estimated during a calibration phase using dedicated pilot symbols.

\section{ERSM PERFORMANCE AND OPTIMUM POWER RATIO}

\section{A. Bit Error Probability performance}

In this section, we provide the performance analysis of the ERSM scheme by deriving the Bit Error Rate (BER) defined by $\mathcal{P}_{e}$. A perfect channel estimation at the transmitter and synchronization are supposed. Let $\mathcal{P}_{S S}$ be the probability of error for the spatial symbol, and $\mathcal{P}_{P S K}$ the average BER of the M-PSK modulation. Each RA has an equal probability of being targeted by $P_{1}$ or $P_{2}$, so the total BEP becomes:

$$
\mathcal{P}_{e}=\frac{\left(\beta_{s} \mathcal{P}_{S S}+\beta_{M} \mathcal{P}_{P S K}\right)}{\beta}
$$

Let $\mathcal{P}_{P S K_{k}}$ be the BER of the M-PSK modulation when sending the spatial symbol $k \cdot \mathcal{P}_{P S K_{k}}$ can be approximated by [17]:

$$
\begin{aligned}
\mathcal{P}_{P S K_{k}} & \approx \frac{1}{\beta_{M}} Q\left(\sqrt{\frac{E_{s}}{N_{0}}} \sin (\pi / M)\right) \\
& =\frac{1}{\beta_{M}} Q\left(\frac{\sum_{j=1}^{N_{r}} \sqrt{p_{j, k}} f \sin (\pi / M)}{\sqrt{N_{r}} \sigma_{n}}\right)
\end{aligned}
$$

where $Q($.$) denotes the Gaussian q-function, E_{s}$ is the symbol energy, and $N_{0}$ the noise spectral density. Let $N_{k}$ be the number of RAs that are targeted with power level $P_{2}$, i.e. the spatial bit associated is "1", we have:

$\mathcal{P}_{P S K_{k}}=\frac{1}{\beta_{M}} Q\left(\frac{\left(N_{r} \sqrt{P_{1}}+N_{k}\left(\sqrt{P_{2}}-\sqrt{P_{1}}\right)\right) f \sin \left(\frac{\pi}{M}\right)}{\sqrt{N_{r}} \sigma_{n}}\right)$

All spatial symbols are equally likely to be sent, so the average BEP of the MPSK symbol is:

$$
\mathcal{P}_{P S K}=\frac{1}{2^{N_{r}}} \sum_{N_{k}=0}^{N_{r}} C_{N_{r}}^{N_{k}} \mathcal{P}_{P S K_{k}}
$$

On the other hand, the probability of error in the spatial bit $\mathcal{P}_{S b}$ is:

$$
\begin{aligned}
\mathcal{P}_{S b}=\frac{1}{2} \mathcal{P} & \left(\sqrt{P_{1}} b_{m_{j}} \rightarrow \sqrt{P_{2}} b_{m_{j}}\right) \\
& +\mathcal{P}\left(\sqrt{P_{2}} b_{m_{j}} \rightarrow \sqrt{P_{1}} b_{m_{j}}\right)
\end{aligned}
$$

where $\mathcal{P}(x \rightarrow y)$ is the probability to send $x$ and detect $y$.

Then, after applying the threshold detection, and supposing that the PSK symbol is well estimated, we obtain:

$$
\begin{aligned}
\mathcal{P}_{S b} & =\frac{1}{2} \times\left[\mathcal{P}\left(\nu \leq \Re\left\{f \sqrt{p_{1}} b_{m_{j}}+\eta_{j}\right\}\right)\right. \\
& \left.+\mathcal{P}\left(\Re\left\{f \sqrt{p_{2}} b_{m_{j}}+\eta_{j}\right\} \leq \nu\right)\right]
\end{aligned}
$$

and after mathematical derivations, we get:

$$
\mathcal{P}_{S b}=Q\left(\frac{f \cdot\left(\sqrt{P_{2}}-\sqrt{P_{1}}\right)}{2 \sigma_{n}}\right)
$$

In fact, since the compensation of the phase is needed before the spatial detection, it seems that the PSK demodulation affects in the spatial detection. But for high SNR, the effect is negligible, and it is evident then to take the approximation of the performance supposing that the phase is well estimated. The BER of the whole spatial symbol is given as:

$$
\mathcal{P}_{S S}=\frac{1}{N_{r}} \sum_{j=1}^{N_{r}} \mathcal{P}_{S b}
$$

We can notice that assuming a perfect channel estimation at the transmitter for the pre-processing, the same signal to noise ratio for each RA and decorrelation between these RA, we can straightforwardly get:

$$
\mathcal{P}_{S S}=\mathcal{P}_{S b}
$$

\section{B. Optimal Power Ratio $\alpha$}

The optimal value of $\alpha$ has to deal with the difference between two power levels to minimize the error in the spatial detection, and should satisfy a sufficient signal-to-noise ratio for the symbol transmitted with the lower power level summation, i.e. when all RAs are targeted with $P_{1}$, in order to minimize the error in the M-PSK demodulation.

Define $f_{S}$ as:

$$
f_{S}(\alpha)=\frac{f}{2 \sigma_{n}}\left(\sqrt{P_{1}}-\sqrt{P_{2}}\right)=\frac{f}{2 \sigma_{n}} \frac{1-\sqrt{\alpha}}{\sqrt{1+\alpha}}
$$

and $f_{M}$ as:

$$
f_{M}(\alpha)=\frac{\sqrt{N_{r}} f}{\sigma_{n}} \sqrt{P_{1}} \sin (\pi / M)=\frac{f}{\sigma_{n}} \frac{\sqrt{N_{r}} \sin (\pi / M)}{\sqrt{1+\alpha}}
$$

as the functions that are taken from the error probability of equations (24) and (19) respectively. So we have:

$$
\alpha_{o p t}=\arg \max _{\alpha}\left\{\min \left\{f_{S}(\alpha), f_{M}(\alpha)\right\}\right\}
$$

that becomes:

$\alpha_{o p t}=\arg \max _{\alpha}\left\{\min \left\{\frac{f}{2 \sigma_{n}} \frac{1-\sqrt{\alpha}}{\sqrt{1+\alpha}}, \frac{f}{\sigma_{n}} \frac{\sqrt{N_{r}} \sin (\pi / M)}{\sqrt{1+\alpha}}\right\}\right\}$.

This optimization equation is equivalent to finding the value of $\alpha$ that makes the two terms equal, which gives:

$$
\alpha_{o p t}=\left(1+2 \sqrt{N_{r}} \sin (\pi / M)\right)^{2}
$$

This optimal value will be compared with the value obtained by simulations in section $\mathrm{V}$. 


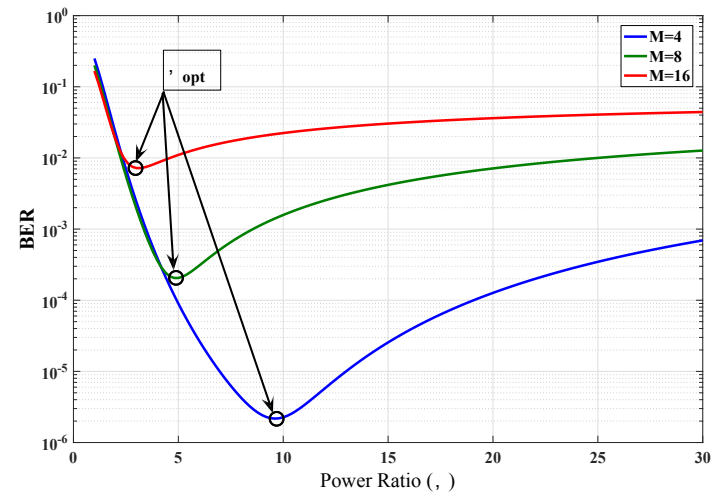

Figure 3. BER vs power ratio $\alpha$ for a $8 \times 2$ MIMO with ERSM QPSK, 8-PSK and 16-PSK

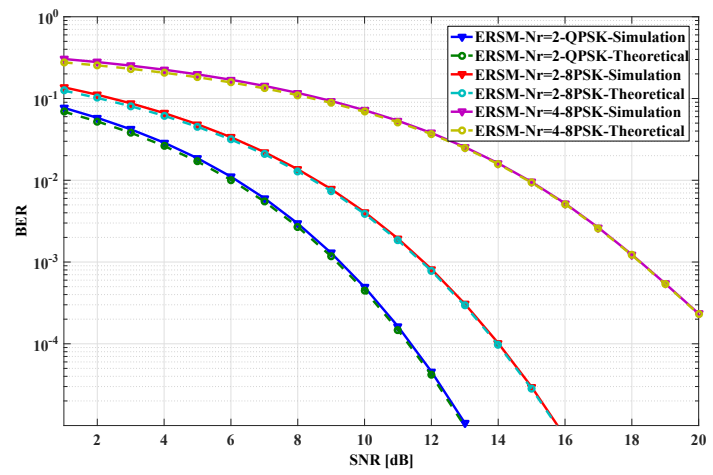

Figure 4. Theoretical and Simulation BER results for $8 \times 2$ and $8 \times 4$ ERSM with QPSK and 8-PSK, with Rayleigh fading.

\section{Simulation Results}

The performance of the proposed ERSM scheme is evaluated through the measurement of the BER versus the ratio between the average transmit power level and noise level, i.e. $\frac{\bar{P}_{t}}{\sigma_{n}^{2}}$ defined as the transmitted signal power to noise ratio (SNR). It is assumed that $\mathbf{H}$ is a MIMO flat fading channel matrix where $H_{j, i}$ are complex coefficients following i.i.d. Rayleigh distribution. The power for each sub-channel is normalized:

$$
E\left[\left\|H_{j, i}\right\|^{2}\right]=1
$$

Finally, we consider that the channel response is perfectly known at the transmitter, so that perfect ZF pre-processing is performed.

Simulations are run by implementing a sufficient number of iterations for different channel realizations and taking the mean value of the BER for each SNR value.

In Fig. 3, the performance of ERSM system with $N_{r}=2$ and $N_{t}=8$ is provided as function of the power ratio $\alpha$. A minimum of BER is reached at the point of the optimal value of $\alpha$ that was analytically calculated in the previous part. In later simulations, the optimal value of alpha is taken to choose the power levels.

In Fig. 4, we compare the simulation results with the theoretical results provided by the derivation of the theoretical

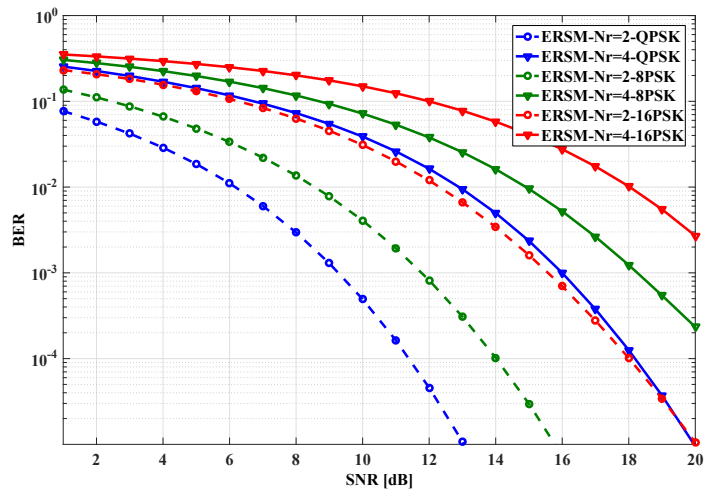

Figure 5. BER vs SNR for a $8 \times 2$ and $8 \times 4$ MIMO ERSM, for QPSK, 8-PSK, and 16-PSK with Rayleigh fading.

analysis of the BER. Three ERSM with $N_{t}=8$ TAs and with different configurations regarding $N_{r}$ and the order of IQ modulation are provided: ERSM-QPSK with $N_{r}=2$, ERSM-8PSK with $N_{r}=2$, and ERSM-8PSK with $N_{r}=4$. Results obviously show that the simulation results perfectly match with the theoretical derivation for high SNR, and with a negligible difference in low SNR.

In Fig. 5, the performance of the ERSM system when increasing the SE by increasing the order of spatial modulation, i.e. $N_{r}$, or by increasing the order of IQ modulation. Recall that the spectral efficiency of the system is $N_{r}+\log _{2}(M)$, we provide different ERSM systems with $N_{t}=8, N_{r}=2$ or 4, and with $M=4,8$ and 16 for each configuration. Result show obviously that increasing the SE, whether by increasing $N_{r}$ or $M$ leads to a degradation in performance. But when focusing on the same SE but different system configuration, i.e. for $N_{r}=2$ with 16PSK, and $N_{r}=4$ with QPSK modulation, the $\mathrm{SE}=5 \mathrm{bit} / \mathrm{s} / \mathrm{Hz}$ for each, it is shown that the performance is approximately the same especially when increasing the SNR. This result is because of the choosing of the optimal solution, since for the higher SNR, the dominant error in the IQ symbols results from the symbol with the lower power (i.e. when the spatial symbol is all zeros), and we demonstrate in the analytical part that the optimal ratio results if the error in the spatial modulation is equal to the error resulted from the IQ symbol with the minimum power.

\section{CONCLUSION}

In this paper, we proposed a very efficient way to improve the spectral efficiency related to the space dimension of spatial modulations at the receiver side, by sending an additional Mary symbol to the extended space shift keying. The introduced ERSM system targets all receive antennas by two different power levels, in order to transmit spatial and M-ary symbols. Consequently, ERSM allows the transmission of $N_{r}+\log (M)$ bits during each symbol duration. We analytically derived an optimal value for the power level ratio that was further validated by simulations. We also expressed the analytical BER performance for the system that was compared and showed to match the simulation results. Moreover, simulations show 
that when using the optimal power ratio, the performance of the ERSM system can be the same when variating the spatial modulation order or the IQ modulation order for the same spectral efficiency.

\section{ACKNOWLEDGMENT}

The authors would like to thank the SPATIAL MODULATION project funded by the French National Research Agency (ANR).

\section{REFERENCES}

[1] G. Tsoulos, Mimo system technology for wireless communications. CRC press, 2006.

[2] D. Gesbert, M. Kountouris, R. W. Heath Jr, C.-B. Chae, and T. Salzer, 'Shifting the mimo paradigm', IEEE signal processing magazine, vol. 24, no. 5, pp. 36-46, 2007.

[3] Y. A. Chau and S.-H. Yu, 'Space modulation on wireless fading channels', in Vehicular Technology Conference, 2001. VTC 2001 Fall. IEEE VTS 54th, IEEE, vol. 3, 2001, pp. 1668-1671.

[4] J. Jeganathan, A. Ghrayeb, L. Szczecinski, and A. Ceron, 'Space shift keying modulation for mimo channels', IEEE Transactions on Wireless Communications, vol. 8, no. 7, pp. 3692-3703, 2009.

[5] R. Y. Mesleh, H. Haas, S. Sinanovic, C. W. Ahn, and S. Yun, 'Spatial modulation', IEEE Transactions on Vehicular Technology, vol. 57, no. 4, pp. 2228-2241, 2008.

[6] A. Younis, N. Serafimovski, R. Mesleh, and H. Haas, 'Generalised spatial modulation', in 2010 Conference Record of the Forty Fourth Asilomar Conference on Signals, Systems and Computers, IEEE, 2010, pp. 1498-1502.

[7] L.-L. Yang, 'Transmitter preprocessing aided spatial modulation for multiple-input multiple-output systems', in Vehicular Technology Conference (VTC Spring), 2011 IEEE 73rd, IEEE, 2011, pp. 1-5.

[8] A. Stavridis, S. Sinanovic, M. Di Renzo, and H. Haas, 'Transmit precoding for receive spatial modulation using imperfect channel knowledge', in Vehicular Technology Conference (VTC Spring), 2012 IEEE 75th, IEEE, 2012, pp. 1-5.

[9] D.-T. Phan-Huy and M. Hélard, 'Receive antenna shift keying for time reversal wireless communications', in 2012 IEEE International Conference on Communications (ICC), IEEE, 2012, pp. 4852-4856.

[10] A. Mokh, Y. Kokar, M. Hélard, and M. Crussière, 'Time reversal receive antenna shift keying on mimo los channel', in 2017 International Conference on Sensors, Networks, Smart and Emerging Technologies (SENSET), IEEE, 2017.

[11] R. Zhang, L.-L. Yang, and L. Hanzo, 'Generalised precoding aided spatial modulation', IEEE Transactions on Wireless Communications, vol. 12, no. 11, pp. 54345443, 2013.
[12] A. MOKH, M. Hélard, and M. Crussière, 'Extended receive antenna shift keying', in 2017 IEEE International Conference on Telecommunication (ICT), IEEE, 2017.

[13] A. Mokh, M. Hélard, and M. Crussière, 'Space shift keying modulations for low complexity internet-ofthings devices', in 2017 Global Communications Conference (GLOBECOM), IEEE, 2017.

[14] A. Mokh, M. Crussière, and M. Hélard, 'Performance analysis of the maximum ratio transmission preprocessing for extended receive antenna shift keying', in 2017 International Symposium on Wireless Personal Multimedia Communications (WPMC), IEEE, 2017.

[15] — 'Performance analysis of extended rask under imperfect channel estimation and antenna correlation', in 2018 IEEE Wireless Communications and Networking Conference (WCNC), IEEE, 2018.

[16] C. Masouros and L. Hanzo, 'Dual-layered mimo transmission for increased bandwidth efficiency', IEEE Transactions on Vehicular Technology, vol. 65, no. 5, pp. 3139-3149, 2016.

[17] J. G. Proakis, 'Digital communications. 1995', McGraw-Hill, New York, 